\title{
ACESSO SUB-FRONTAL A LESÕES SELARES E PARA-SELARES
}

\author{
JOSÉ ALBERTO LANDEIRO*, MARLO STEINER FLORES**, CARLOS ANDRÉ R. LOPES ***, \\ MÁRIO ALBERTO LAPENTA***, CARLOS HENRIQUE RIBEIRO****
}

\begin{abstract}
RESUMO - Os processos expansivos situados ao nível da região selar constituem um variável número de patologias que, apesar da localização semelhante, têm características clínicas, radiológicas, patológicas e origens diferentes. Assim sendo, diferentes tipos de abordagens são utilizados no tratamento cirúrgico destas lesões. Dentre os acessos transcranianos convencionais e os mais sofisticados acessos à base do crânio, o acesso sub-frontal proporciona visão direta das estruturas neurovasculares da região. Este estudo descreve 19 tumores operados por esta via, no período compreendido de fevereiro de 1997 a março de 1998. Onze destas lesões eram adenomas pituitários, 3 eram craniofaringiomas, 2 eram meningiomas, 1 germinoma e 1 lesão inflamatória do nervo óptico. A remoção total dos tumores foi conseguida em 17 casos. Não houve mortalidade operatória e as complicações mais comuns foram: anosmia unilateral, que ocorreu em 12 casos; diabetes insipidus transitório, que acometeu 4 pacientes; e déficit isquêmico progressivo, que ocorreu em 1 paciente. Estes resultados demonstram que a via sub-frontal é uma opção segura para a cirurgia dos tumores desta região.
\end{abstract}

PALAVRAS-CHAVE: acesso sub-frontal, tumor selar, expansão supra-selar.

\section{Subfrontal approach in sellar surgery}

ABSTRACT - A variety of mass lesions are placed in and around of the sella turcica. Although their common location these lesions have distinguishing features and different approaches can be selected. There are conventional transcranial and sophisticated skull base approaches. We report our experience with a subfrontal route to the sellar region. Between February 1997 and March 1998, 19 tumors placed around the sella have been treated surgically by this method. Eleven of them were pituitary tumors, 3 were craniopharyngeomas, 2 were meningiomas, and 1 was germinoma. Total removal was achieved in 17 cases. There was not operative mortality. Postoperative complications included twelve cases of unilateral anosmia and four cases of transitory diabetes insipidus. Progressive stroke occurred in one patient. These results stress the importance of the subfrontal approach to reach such lesions in sellar region.

KEY WORDS: subfrontal approach, sellar region tumor, suprasellar extension.

As lesões situadas ao nível da região selar sejam elas localizadas no interior da sela, para ou supra-selares representam enorme número de patologias ${ }^{1}$, as quais são operadas por diferentes vias de acesso. Entre estas destacam-se as vias pterional, bifrontal inter-hemisférica, fronto-órbitozigomática e suas variantes, trans-naso-esfenoidal e a via sub-frontal, uma das mais antigas ${ }^{2-7}$. Com o desenvolvimento das técnicas de cirurgia da base do crânio, a via sub-frontal foi praticamente descartada. $\mathrm{O}$ maior motivo era a excessiva retração cerebral e a presença da borda orbitária, uma barreira óssea que se interpõe entre a visão do neurocirurgião e o campo operatório ${ }^{8}$.

Serviço de Neurocirurgia do Hospital de Força Aérea do Galeão (HFAG): *Chefe do Serviço de Neurocirurgia do HFAG, Professor Assistente-Doutor do Hospital Universitário Antônio Pedro, Professor convidado do Hospital Universitário Pedro Ernesto; **Chefe de Clínica do Serviço de Neurocirurgia do HFAG; ***Neurocirurgião Assistente do Serviço de Neurocirurgia do HFAG. Aceite: 5-outubro-1999.

Dr. José Alberto Landeiro - Estrada do Galeão 4101, Ilha do Governador - 21941-000 Rio de Janeiro RJ - Brasil. FAX 21284 5341. E-mail: msteiner@unisys.com.br 
Com maior conhecimento da microanatomia da região, bem como dos grandes avanços e refinamentos nas técnicas de neuroanestesia e microcirurgia, a via sub-frontal volta a ser considerada uma opção para tratar as lesões selares e paraselares?.

Este estudo discute as possibilidades do emprego da via sub-frontal na cirurgia de lesões selares e para-selares, ao analisar uma série de pacientes operados nos últimos 12 meses.

\section{MÉTODO}

Dezenove pacientes portadores de diversas lesões peri-selares foram operados. A idade do grupo variou entre 12 e 64 anos com média de 37 anos. Onze pacientes eram do sexo feminino e 8 do sexo masculino. Dezessete pacientes foram operados no Hospital de Força Aérea do Galeão e 2 no Hospital Universitário Antônio Pedro. Todos os pacientes foram submetidos a tomografia computadorizada (TC) e ressonância magnética (RNM), exames bioquímicos, hormonais, avaliação imuno-histoquímica no caso de lesões pituitárias não-funcionantes e avaliação neuro-oftalmológica, que constou de campimetria e acuidade visual. Vale ressaltar que os 11 tumores pituitários (Tabela 1) foram operados como parte de uma estratégia de cirurgia em 2 estágios, isto é, a via transesfenoidal foi a primeira opção cirúrgica para o tratamento destes tumores e, caso a lesão não fosse removida totalmente, o paciente seria operado pela via sub-frontal.

\section{Técnica cirúrgica}

O paciente é colocado em decúbito dorsal com a cabeça rodada no máximo até $20^{\circ}$, ligeiramente estendida, de modo a que a proeminência do malar fique no ponto mais alto em relação ao campo operatório. A incisão do couro cabeludo é semi-coronal, por trás da linha do cabelo estendendo-se até a projeção da linha médio-pupilar contralateral, evitando-se assim tração desnecessária do retalho cutâneo. A craniotomia (realizada com craniótomo da 3M ou Ultra Power Drill System da Zimmer) constou de orifício de trepanação atrás do zigoma, acima da junção fronto-esfenoidal. Este detalhe anatômico é obtido com a desinserção de aproximadamente 1 a $2 \mathrm{~cm}$ do músculo temporal da linha curva temporal. A craniotomia é paralela e rasante à borda orbitária superior estendendose até $1 \mathrm{~cm}$ da linha média, a maioria das vezes abrindo-se o seio frontal. A partir deste ponto o craniótomo descreve um arco afastando-se da linha média, com raio de aproximadamente $3 \mathrm{~cm}$ até encontrar o orifício inicial (Fig 1). Antes da abertura da duramáter, a mucosa do seio frontal é removida e o seio é tamponado com cotonóide. A duramáter é incisada paralelamente à borda orbitária e refletida em direção à base. Após a remoção da lesão, o seio frontal é ocluido com retalho de gálea, a qual é suturada à duramáter. O retalho ósseo é fixado com miniplacas de titânio ou com fio Prolene - 0 .

\section{RESULTADOS}

Do total de 19 pacientes, 11 apresentavam adenomas pituitários já previamente operados pela via transesfenoidal e com laudo estabelecido (Tabela 2). A Tabela 3 relaciona os sinais e sintomas mais comumente encontrados. Sinais e sintomas relacionados a hipopituitarismo, comprovado laboratorialmente, ocorreram em 9 pacientes. Duas pacientes apresentaram quadro clínico de diabetes insipidus, uma delas apresentando síndrome de galactorréia - amenorréia (Tabela 1).

Tabela 1. Tipos de patologias operadas pela via sub-frontal lateral.

\begin{tabular}{lc}
\hline Tipos de patologias & $\mathrm{N}^{0}$ de pacientes \\
\hline Adenoma pituitário & 11 \\
Craniofaringioma & 3 \\
Tumor hipotalâmico (granuloma eosinofílico) & 1 \\
Processo expansivo do nervo óptico (granulomatose de Wagener) & 1 \\
Meningioma do tubérculo selar & 1 \\
Meningioma do diafragma selar & 1 \\
Germinoma supra-selar & 1 \\
\hline
\end{tabular}


Tabela 2. Sinais e sintomas no pré-operatório.

\begin{tabular}{lc}
\hline Sinais e sintomas & $\mathrm{N}^{0}$ de pacientes \\
\hline Cefaléia & 17 \\
Déficit de campo visual & 10 \\
Pan-hipopituitarismo & 9 \\
Déficit de acuidade visual & 7 \\
Paresia do 3 $3^{\circ}$ nervo craniano & 3 \\
Diabetes insipidus & 2 \\
Galactorréia - amenorréia & 1 \\
\hline
\end{tabular}

A remoção total foi obtida em 17 casos e, em uma paciente com lesão hipotalâmica, conseguiuse apenas a remoção parcial (Figs 2 e 3). Em uma outra que apresentava processo expansivo do nervo óptico, durante a cirurgia constatou-se processo inflamatório, cuja biopsia revelou tratar-se de granulomatose de Wegener.

Após a cirurgia, dos 7 pacientes com déficit de acuidade visual, 5 melhoraram e 2 permaneceram inalterados; dos 10 pacientes com déficit de campo visual, em 7 ocorreu a recuperação e 3 permaneceram com as mesmas alterações.

A cirurgia estabilizou o quadro de déficit hormonal em 5 pacientes que continuam realizando terapia de reposição e em 4 houve normalização das taxas hormonais. Dois pacientes com acromegalia tiveram normalização das taxas de GH (hormônio do crescimento) e do IGF-1. Uma paciente evoluiu a partir do $4^{0}$ dia de pós-operatório com progressivo déficit motor e a TC de crânio revelou àrea de infarto isquêmico nos lobos frontal, temporal e parietal. Tratava-se de meningioma clinoidal com envolvimento da artéria carótida na sua porção supraclinóidea, e da emergência das artérias cerebral anterior e média. Provavelmente a extensa dissecção do tumor ao nível da parede das artérias ocasionou um vasoespasmo generalizado.

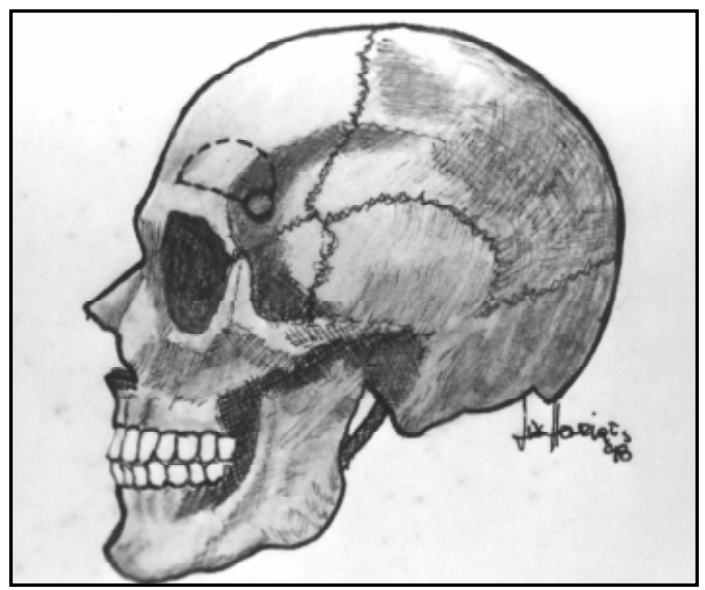

Fig 1. Acesso sub-frontal. 


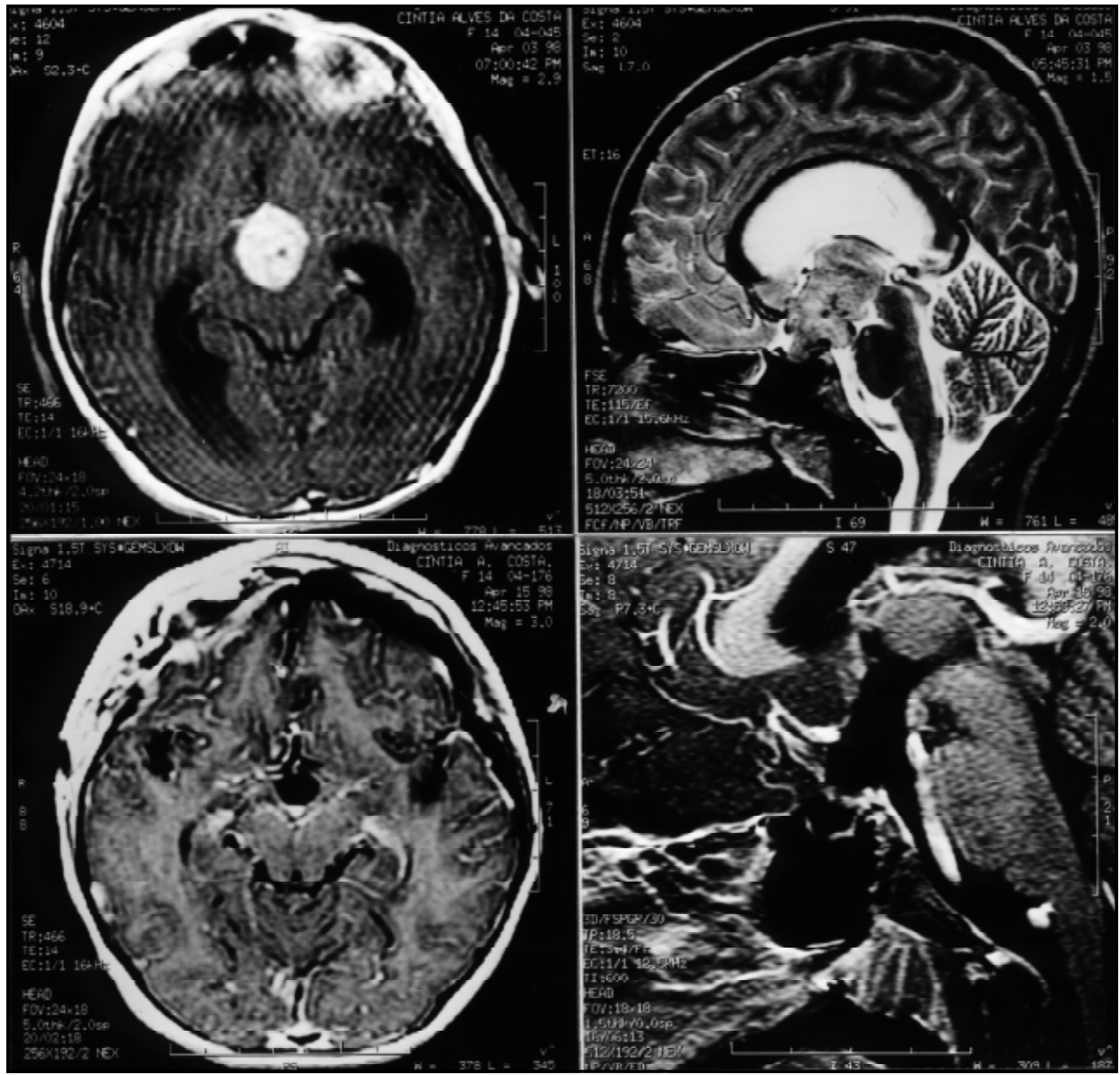

Fig 2. RM em corte sagital e coronal mostrando volumoso processo expansivo preenchendo a porção anterior do $3^{o}$ ventrículo $(A$ e $B$ ). As imagens no pós-operatório ( $C$ e D) mostram remoção completa da lesão. $O$ laudo histopatológico foi de germinoma.

A preservação unilateral do nervo olfatório foi conseguida em 9 pacientes e bilateralmente em 2, porém a função olfatória foi preservada em 7 . Em 2 pacientes o quiasma era pré-fixado e, em ambos a remoção do tumor foi feita através do espaço inter-óptico-carotídeo direito.

Em 4 pacientes os tumores apresentavam-se com expansão lateral direita e a lesão foi removida através de craniotomia sub-frontal esquerda. O restante das lesões era de linha média e os pacientes foram operados através do acesso sub-frontal direito com remoção inter-óptico-quiasmática.

Não houve mortalidade operatória. Um paciente portador de gonadotrofinoma invasivo faleceu 6 meses após a cirurgia por recidiva da lesão, apresentando erosão da base do crânio, infiltração hipotalâmica e fístula liquórica. Outra paciente com 13 anos, portadora de germinoma supra-selar, que teve a lesão removida totalmente, faleceu de septicemia 2 meses após a cirurgia, na vigência de quimioterapia.

\section{DISCUSSÃO}

O desenvolvimento das técnicas da cirurgia da base do crânio visa proporcionar ao neurocirurgião menor ou nenhuma retração cerebral, melhorar a iluminação, e com isto obter um 


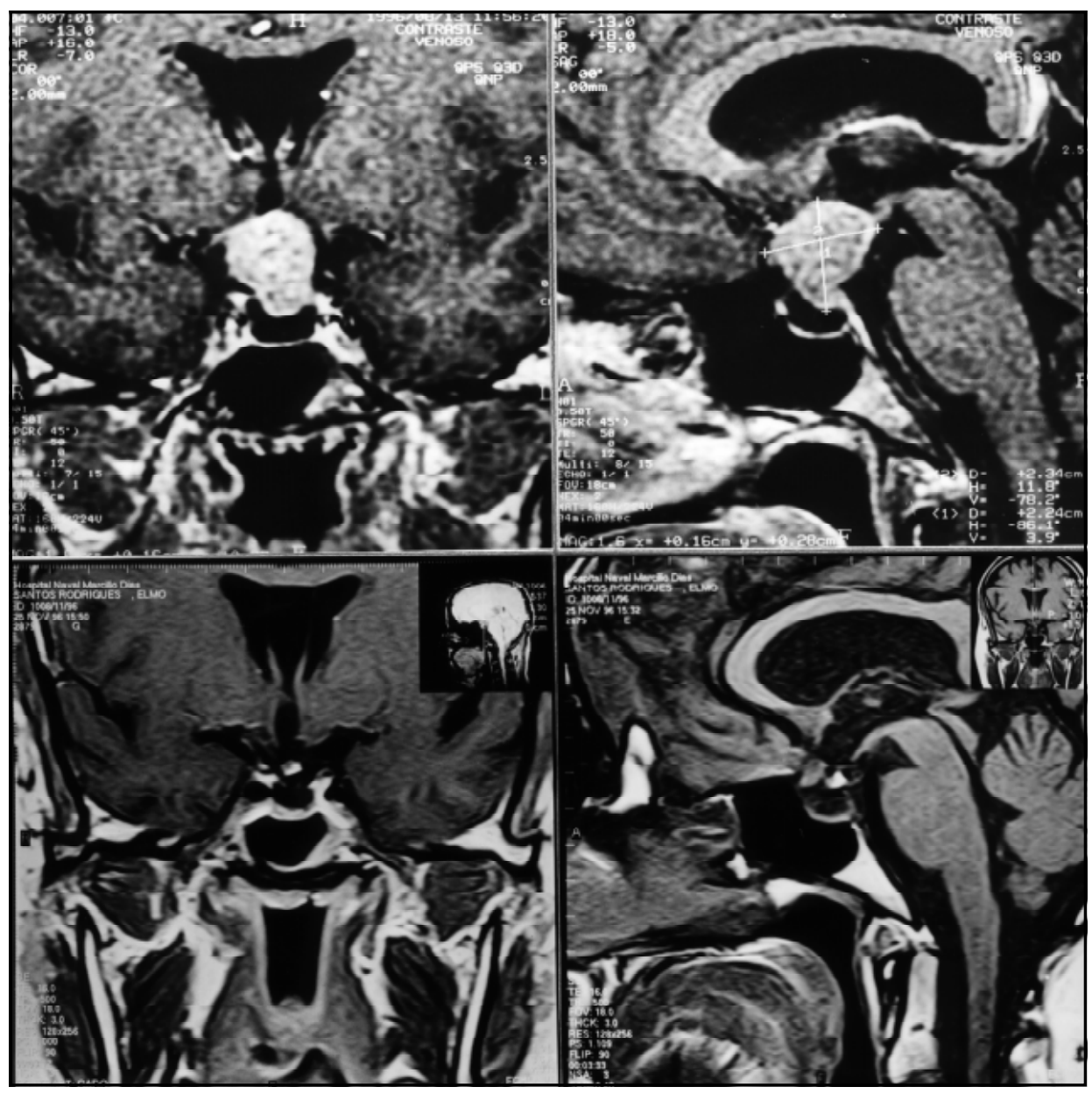

Fig 3. RM mostrando lesão com sinal hiperintenso e captante de contraste, penetrando no interior da sela (A e B). No pós-operatório imagens ( $C$ e D) mostrando ressecção total da lesão. Tratava-se de meningioma do diafragma selar.

total controle das estruturas neurovasculares envolvidas na cirurgia. Para isto, sofisticadas vias de acesso, que requerem dispendioso arsenal de instrumentos, são hoje de domínio dos neurocirurgiões. Todas elas convergem em um ponto: remover estruturas ósseas do modo mais anatômico possível e reconstrui-las, sem trazer nenhum defeito cosmético ou funcional.

Os tumores localizados ao nível da região selar têm diferentes origens e como tal devem ser tratados ${ }^{3}$. Entre as opções de abordagens transcranianas destacam-se as vias pterional e sub-frontal. A via pterional, do domínio da maioria dos neurocirurgiões e a mais amplamente utilizada, e a via sub-frontal, a mais antiga, deram origem a inúmeras variantes como: fronto-orbital, fronto-orbitozigomática, sub-temporal com ressecção do arco zigomático ${ }^{2,4,10-13}$. A via pterional, tal como idealizada por Yasargil ${ }^{6}$, além de ser o acesso preferencial para abordar a quase totalidade dos aneurismas intracranianos, também é proposta para tratar processos expansivos ao nível da região selar, parasselar e terço superior do clivus ${ }^{7}$. O mesmo autor cita o acesso pterional, ao operar com sucesso cistos epidermóides e dermóides, ao nível das cisternas temporo-basais ${ }^{7}$. A via sub-frontal leva-nos ao princípio do século, quando Frazier ${ }^{5}$ a indicou para acesso aos tumores da hipófise. Jefferson ${ }^{14}$, em 
1953, ao apresentar no Royal College of London notável trabalho sobre tumores pituitários, propôs a via sub-frontal como eletiva para tratar as expansões supra-selares. Ao longo dos anos, a via subfrontal foi largamente utilizada por diversos autores para tratar lesões como craniofaringiomas e outras lesões não pituitárias da região ${ }^{15,16}$.

A excessiva retração cerebral fez autores como Jane e col. ${ }^{10}$ proporem a modificação da via, a qual constou da remoção da borda orbitária. Tal proposta foi não só seguida, como algumas modificações realizadas, todas elas com a finalidade de aumentar o campo operatório, sem retrair o cérebro $^{2,4,11,12,17}$.

Alertado pelos trabalhos de Hoffman e Raffel ${ }^{15}$, além de Samii ${ }^{9}$, que preconizam o acesso sub-frontal simples para tumores tipo craniofaringeomas e outras lesões volumosas da região, inclusive com ocupação do $3^{\circ}$ ventrículo, resolvemos reativar a via no Serviço.

As novas drogas anestésicas, além dos novos e seguros aparelhos utilizados em anestesia, permitem que com leve retração possamos facilmente atingir a cisterna quiasmática, obtendo drenagem liquórica e com isso aumentando o relaxamento cerebral. Além disso, há possibilidade de abrir-se a cisterna de Sylvius já ao nível de sua porção pterional. Por último, a própria lesão tumoral causa o deslocamento do cérebro e com isto cria o espaço necessário para sua remoção. Tais fatos, acrescidos de inúmeros tumores da hipófise necessitando de uma segunda abordagem, nos fez adotar este procedimento. A indicação da via e a estratégia cirúrgica dependem da origem, da expansão do tumor, suas relações neurovasculares, se preenche ou não a cavidade do $3^{\circ}$ ventrículo e da posição do quiasma, isto é, se pré ou pós fixado. Um quiasma pré-fixado encobre a porção central do tumor obrigando o neurocirurgião a adotar rotas alternativas. A escolha do lado da craniotomia em lesões situadas na linha média depende do cirurgião. Habitualmente, o cirurgião dextro o faz pela direita. Quando existe expansão lateral, optamos pela craniotomia contralateral, a qual permite melhor visão e controle sub-quiasmal da lesão.

No caso das lesões selares, alguns espaços se apresentam para a ressecção do tumor. A maioria dos tumores da área, como os meningiomas do tubérculo da sela, adenomas pituitários e craniofaringiomas, desloca os nervos ópticos lateralmente e o quiasma para trás, ampliando-se o espaço chamado inter-óptico-quiasmatico, por onde estes tumores são removidos. Um outro espaço, que quando amplo pode ser usado para abordar lesões com projeção mais posterior e em pacientes com quiasma pré-fixado, é o interóptico-carotídeo.

O espaço lateral à carótida representa uma abordagem adicional às lesões com expansão lateral e que comprimem o $3^{\circ}$ nervo craniano. Esta rota é adotada naqueles casos em que a lesão ocupa a porção anterior do $3^{\circ}$ ventrículo ou quando a lesão não é acessível pela via inter-ópticoquiasmática, como nos casos de quiasma pré-fixado.

A remoção das lesões selares tem índice extremamente elevado de complicações endócrinas, principalmente diabetes insípidus ${ }^{18}$. Tal fato é, inclusive, aceito como ocorrência comum nas remoções totais dos craniofaringiomas ${ }^{19}$. $\mathrm{O}$ nosso índice de diabetes insipidus foi extremamente baixo. Isto pode ser explicado pelo pequeno número de craniofaringiomas na nossa série, e porque a maioria das lesões deslocavam o talo pituitário para baixo e para trás. Uma cuidadosa remoção intracapsular permitiu a preservação desta estrutura anatômica.

A preservação do olfato deve ser uma preocupação constante dos neurocirurgiões ${ }^{9}$. Os volumosos tumores da região selar elevam e deslocam lateralmente os nervos olfativos. Nestes casos sempre existe um plano de clivagem formado pela aracnóide, o tumor e o nervo, tornando-se possível a preservação anatômica. Porém, nos casos de tumores menores, a abertura das cisternas e o grande espaço criado entre a base do lobo frontal e o assoalho da fossa craniana, facilita a ruptura do nervo no lado da dissecção. A preservação do nervo do outro lado fez com que a maioria dos pacientes não referisse anormalidade com relação ao olfato (Tabela 3). 
Tabela 3. Complicações pós-operatórias.

\begin{tabular}{lc}
\hline Complicações & $\mathrm{N}^{\mathrm{o}}$ de pacientes \\
\hline Anosmia unilateral & 12 \\
Diabetes insipidus transitório & 4 \\
Diabetes insipidus permanente & 3 \\
Anosmia bilateral & 1 \\
Isquemia tardia & 1 \\
Fístula liquórica nasal & 1 \\
Paresia transitória do $3^{\circ}$ nervo craniano & 1 \\
\hline
\end{tabular}

A realização de cirurgias na região selar, que apresenta tênues limites com os seios esfenoidal e etmóide, predispõe ao desenvolvimento de fístula liquórica e consequente infecção. Assim sendo, já na abordagem o neurocirurgião deverá estar atento a esta possibilidade e com isto expor extenso retalho da aponeurose epicraniana com sua fascia célulo adiposa. O retalho deverá ter o pedículo distal junto à órbita e a sua extensão suficiente para cobrir todo o assoalho da fossa craniana anterior e a sela. A fixação do retalho ósseo com microplacas de titânio provê excelente resultado estético.

Em suma, os resultados obtidos nesta série representam um endosso a uma antiga, porém segura e eficaz abordagem craniana. As novas drogas anestésicas e técnicas microcirúrgicas fazem desta via uma valiosa opção para tratar as lesões expansivas ao nível da sela turca.

\section{REFERÊNCIAS}

1. Yamada S. The reverse hemolytic plaque assay. Endocr Pathol 1990;1:129-131.

2. Al-Mefty O. Supraorbital pterional approach to skull base lesions. Neurosurgery 1987;21:474-477.

3. Al-Mefty O. Approach selection in juxtasellar surgery. In Salcman M (ed). Surgery of the cranial base. Boston: Kluvier Academic, 1989:57-71.

4. Delashaw JR JB, Tedeschi H, Rhoton AL. Modified supraorbital craniotomy: technical note. Neurosurgery 1992;30:954-956.

5. Frazier $\mathrm{CH}$. An approach to the hypophysis through the anterior cranial fossa. 1913;57:145-150.

6. Yasargil MG. Operative approach interfascial pterional (frontotemporosphenoidal) craniotomy. In Yasargil MG. Microneurosurgery. Stuttgart: Georg Thieme Verlarg 1984;1:215-233.

7. Yasargil MG, Abernathy CD, Sarioglu AC. Microneurosurgical treatment of intracranial dermoid and epidermoid tumors. Neurosurgery 1989;24:561-567.

8. Albin MS, Bunegin L, Dujovny M, Bennett MM, Janetta PJ, Wisotzkey HW. Brain retractor pressure during intracranial procedures. Surg Forum 1975;26:499-500.

9. Samii M. Technical aspects of excision of giant basal tumors with third ventricular involvement. In Apuzzo MLJ. Surgery of the third ventricle. Baltimore: Williams \& Wilkins, 1987:684-697.

10. Jane JA, Park TS, Pobereskin LH, Winn HR, Butler AB. The supraorbital approach: technical note. Neurosurgery 1982;11:537-542.

11. Jane JA, Newman SA. Transcranial orbital surgery. Clin Neurosurg 1995;43:53-71.

12. Pitelli SD, Almeida GM, Nakagawa EJ, Marchese AJT, Cabral ND. Basilar aneurysm surgery: the subtemporal approach with section of the zygomatic arch. Neurosurg 1986;18:125-128.

13. Van Loveren HR, Mahmood A, Liu SS, Gruber D. Innovations in cranial approaches and exposures: anterolateral approaches. Clin Neurosurg 1995;43:44-52.

14. Jefferson G. Extrasellar extension of pituitary adenomas. Proc R Soc Med 1940;33:433-458.

15. Hoffman HJ, Raffel C. Craniopharyngiomas. In McLaurin RL (ed): Pediatric neurosurgery 2.Ed. Philadelphia: Saunders, 1989:399-408.

16. Shillito J Jr. Craniopharyngiomas: the sub-frontal approach, or none at all? Clin Neurosurg, 1980;27:188-205.

17. Sen C, Catalano PJ. Extended sub-frontal approach to the anterior skull base and clivus. In Torrens M, Al-Mefty O, Kobayashi S. Operative skull base surgery. New York: Churchill Livingstone, 1997:57-87.

18. Landeiro JA, Braga FM. Macroadenomas não-funcionantes da hipófise: controle endócrino e oftalmológico. Arq Bras Neurocirurg 1997; 16:61-76.

19. Mohr G, Hardy J, Comtois RS, Beauregard H. Surgical management of giant pituitary adenomas. Can J Neurol Sci 1990;17:62-66. 